\title{
EDITORIAL
}

\section{Pathogenesis of pulmonary arteriovenous malformations: role of hepatopulmonary interactions}

\section{J J Vettukattil}

Heart 2002;88:561-563

\section{Pulmonary arteriovenous malformations-abnormal communications between pulmonary arteries and veins-can lead to serious haemodynamic consequences, predisposing to varying degrees of intrapulmonary shunting, resulting in cyanosis, clubbing, polycythemia, and impaired exercise tolerance}

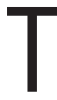
he significance of organ interaction in the pathophysiology of tissue dysfunction is increasingly being recognised as a key determinant influencing resolution of tissue injury. The modulatory effects of such interactions, that incorporate the expanding number of markers of molecular and receptor level cell-to-cell communications, is complex. The liver is a unique organ as it is connected in series with the lung and portal system. It receives all the venous effluent from the portal system and directs its metabolites to the lungs before perfusing any other organ in the body.

Pulmonary arteriovenous malformations (PAVMs) are abnormal communications between pulmonary arteries and veins. They may be hereditary as in Osler-Weber-Rendu disease or acquired as in liver disorders, systemic diseases, venous anomalies, and after palliation of complex cyanotic congenital heart disease. They tend to be progressive and lead to serious haemodynamic consequences, predisposing to varying degrees of intrapulmonary shunting. This results in cyanosis, clubbing, polycythemia, and impaired exercise tolerance. Though hypoxemia and its clinical effects are the main consequences of this condition, serious complications like systemic embolisation, pulmonary haemorrhage, or cerebral abscesses are not uncommon. Evaluation of the underlying pathologic conditions, which predisposes to the development of PAVMs, supports a unifying hypothesis of liver-lung interaction.

\section{HEPATOPULMONARY SYNDROME}

The association of hepatic dysfunction with hypoxaemia in the absence of intrinsic cardiopulmonary disease is known as hepatopulmonary syndrome (HPS). These patients have intrapulmonary vascular dilatations and some develop macroscopic PAVMs. Fluckinger ${ }^{1}$ first described it in 1884 and Kennedy and Knudson ${ }^{2}$ coined the term in 1977. Hepatic dysfunction includes fulminant hepatic failure, cirrhosis, portal hypertension, or rejection of allograft liver transplant. Several mechanisms have been postulated to explain intrapulmonary vascular dilatations in
HPS. These include failure of the damaged liver to clear circulating pulmonary vasodilators, production of a circulating vasodilator or inhibition of a circulating vasoconstrictor by the damaged liver, blunted hypoxic pulmonary vasoconstriction, release of a substance from the diseased liver that promotes fistula formation, and inability of the diseased liver to metabolise various substances present in the portal venous blood. ${ }^{3-6}$

However, conditions with completely normal liver function such as congenital hepatic fibrosis, ${ }^{7}$ portal vein thrombosis, non-cirrhotic portal hypertension, and congenital portovenous shunts also develop PAVMs. Some "unusual" cases of PAVMs reported in the literature in association with carcinoid syndrome also had associated portal vein obstruction. ${ }^{8}$ PAVMs are known to resolve after liver transplantation, further supporting the view that there is a mechanism of liver-lung interaction for the maintenance of normal pulmonary vasculature.

This concept is also supported by the occurrence of PAVMs in a subgroup of patients with palliated complex congenital heart defects.

\section{PAVMS IN PALLIATED CONGENITAL HEART DEFECTS}

Some patients with complex congenital heart defects and univentricular physiology, palliated by connecting the systemic veins directly to the pulmonary circulation, are known to develop PAVMs that are similar to those with hepatic dysfunction (cardiogenic hepatopulmonary syndrome, CHPS). The lungs of such patients are deprived of hepatic venous blood, as in superior cavopulmonary anastomosis or Kawashima operation. It is now well established that even in those patients who do not have macroscopic PAVMs, significant intrapulmonary shunting causing desaturation does occur. ${ }^{9}$ One of the logical explanations is the non-pulsatile nature of the systemic to pulmonary venous connections. However, patients who have undergone long term palliation with Fontan procedures, who share similar haemodynamics but have hepatic veins incorporated in the venous circuit, seldom develop PAVMs. Complete resolution of these malformations occurs after redirection of hepatic veins to the cavopulmonary connection. ${ }^{10}$ This confirms

Abbreviations: CHPS, cardiogenic hepatopulmonary syndrome; HF, hepatic factor(s); HPS, hepatopulmonary sydrome; PAVMs; pulmonary arteriovenous malformations; PVF, portal venous factor(s) 
A

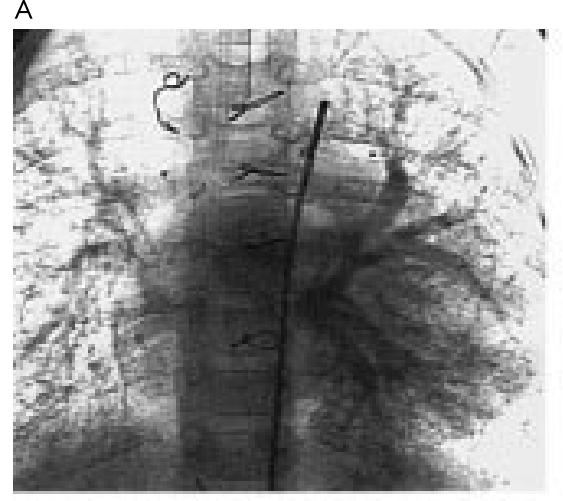

B

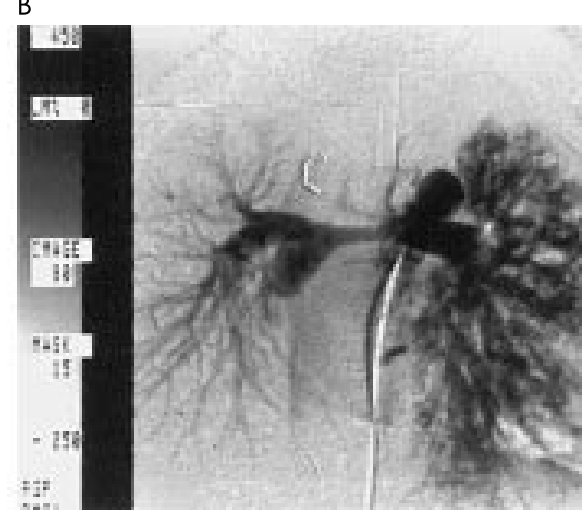

Figure 1 (A) Bilateral diffuse pulmonary arteriovenous malformations in a patient with cardiogenic hepatopulmonary syndrome. (B) Resolution of pulmonary arteriovenous malformations in the right lung and progression in the left lung after redirection of the hepatic veins in the same patient. (Courtesy R Patel, Manchester Children's Hospital) the direct role of hepatic venous effluent in the preservation of the integrity of pulmonary vasculature.

If the pathogenic mechanisms of CHPS and HPS are considered the same, then two possible aetiologic possibilities emerge.

\section{Hepatic factor(s) (HF)}

The reversibility of HPS due to hepatocellular failure by liver transplantation, and CHPS by connecting the hepatic veins into the pulmonary circulation, points to the protective role of metabolites normally present in the hepatic venous blood. Such hepatic factor(s) (HF) have to be removed or significantly reduced on first pass through the circulation to be unavailable in the systemic venous side. It is likely to be a physiologically present constrictor influence. The occurrence of pulmonary hypertension ${ }^{11}$ in a subgroup of patients with advanced liver disease may represent excess production of HF. The role of endothelins in this regard is worth further exploration.

\section{Portal venous factor(s) (PVF)}

Other causes of hepatopulmonary syndrome, where hepatic function is normal and diversion of portal venous flow from the hepatic parenchyma is the dominant feature, suggest the role of dilatory influences present in the portal venous blood which on its own could lead to the development of PAVMs. The portal vein is known to contain vasodilators such as substance P, which are metabolised almost completely by the liver and are increased in other systemic conditions associated with PAVMs.

\section{MECHANISM OF HEPATOPULMONARY INTERACTIONS}

Severe hepatocellular dysfunction would decrease the production of HF and its protective effects on the pulmonary vasculature. This could lead to the development of HPS in fulminant hepatic failure. Obstruction to the portal veins, or portal hypertension associated with porto-systemic shunts, would prevent normal hepatic metabolism of the PVF and diversion into the systemic veins, resulting in increased concentrations of PVF perfusing the pulmonary vascular bed. Coexistence of both hepatocellular injury (decreased production of HF) and portal hypertension (decreased metabolism of PVF), as in the rat bile duct ligation model of HPS ${ }^{12}$ or biliary cirrhosis, would increase the propensity for the development of PAVMs. It is possible that a mechanism of hepatic regulation of pulmonary vascular tone is part of our normal physiology, such that the HF is produced by the hepatic parenchyma under the stimulation of the PVF. The common bile duct ligated rat model of HPS by Luo and group provides further insight into the multifactorial influences in the pathophysiology of liver-lung interaction. ${ }^{13}$

\section{PATHOPHYSIOLOGICAL ASPECTS}

Pathologically, PAVMs are dilated capillary and precapillary vessels associated with evidence of direct arteriovenous communications. ${ }^{14}$ This finding in association with thinning of the vessel wall suggests a process of persistent unopposed vasodilatation rather than angiogenesis as the pathogenic event. Presence of increased concentrations of nitric oxide and constitutive endothelial nitric oxide synthase in the pulmonary arteries of these patients further supports excessive vasodilatory influence as the pathogenic mechanism. ${ }^{12}$ In plexogenic arteriopathy of cirrhosis, vascular deformation caused by medial hypertrophy and fibrosis is combined with dilated thin walled distal channels, perhaps reflecting the end stage of a long battle between constrictor and dilator influences on the pulmonary vasculature. The vascular pathology of pulmonary hypertension seen in $1-2 \%$ of patients with cirrhosis $^{15}$ is indistinguishable from other causes of pulmonary hypertension. In this issue of the journal Ashrafian and Swan are postulating some interesting views regarding the pathogenesis of CHPS. ${ }^{16}$

Careful delineation of the hepatic veins is important in the assessment of all patients with PAVMs. On rare occasions, isolated hepatic venous drainage into the left atrium or bilateral drainage of hepatic veins into right and left atria have been known to result in this condition. Assessing the proximity of the hepatic veins to the azygos or hemiazygos continuation of the inferior vena cava, with a view to incorporate it into the venous circulation at the time of cavopulmonary anastomosis, would prevent the onset of CHPS. In rare cases, diversion of hepatic venous flow from the Fontan circulation into the left atrium through collaterals or associated portal hypertension and congenital or acquired porto-systemic shunts may need to be excluded.

Currently the management of well established PAVMs is limited. Though liver transplantation offers better outcome for HPS patients, the slow regression of these anatomic defects still pose a significant threat in the immediate postoperative period. While redirection of hepatic venous flow in CHPS has shown resolution of PAVMs, associated problems caused by congestive hepatic dysfunction and persistence of malformations in the contralateral lung when preferential blood flow from the hepatic veins is directed to one lung has been observed (fig 1). Experience with inhibition of nitric oxide production using methylene blue is limited ${ }^{17}$ and risk of increasing pulmonary vascular resistance in the setting of Fontan circulation is prohibitive in its routine clinical use.

Further understanding of the pathophysiology of this complex condition and identification of various factors involved are crucial for the prevention and long term management of these patients. The role of endothelins and the effect of its receptor blockers may prove beneficial in future. 


\section{REFERENCES}

1 Fluckinger M. Vorkommen von Trommelschagelformigen fingerend Phalagen ohne chronische Veranderungen an der Lungen oder am Herzen. Wein Med Wochenschr 1884;49:1457.

2 Kennedy TC, Knudsen RJ. Exercise-aggravated hypoxemia and orthodeoxia in cirrhosis. Chest 1977;72:305-9.

3 Zipser RD, Hoefs FS, Speckart PF, et al. Prostaglandins: modulator of renal function and pressor resistance in chronic liver disease. J Clin Endocrinol Metab 1979;48:895-900.

4 Hortnagl $\mathbf{H}$, Singer EA, Lenz K, et al. Substance $P$ is markedly increased in patients with hepatic coma. Lancet 1984;i:480-3.

5 Krowka MJ, Cortese DA. Hepatopulmonary syndrome: an evolving perspective in the era of liver transplantation. Hepatology 1990;1 1:138-42.

6 Gines P, Jimenez W, Arroyo V, et al. Atrial natriuretic factor in cirrhosis with ascites: plasma levels, cardiac release and splanchnic extraction. Hepatology 1988:8:636-42.

7 Maggiore G, Borgna-Pignatti C, Marni E, et al. Pulmonary arteriovenous fistula: an unusual complication of congenital hepatic fibrosis. J Pediatr Gasteroenterol Nutr 1983;2:183-6.

8 Pappagianis J, Kanter RJ, Effman EL, et al. Polysplenia with pulmonary arteriovenous malformations. Pediatr Cardiol 1993;14:127-9.

9 Vettukattil JJ, Slavik Z, Lamb RK, et al. Intrapulmonary arteriovenous shunting, a universal phenomenon in patients with the superior cavopulmonary anastomosis: a radionuclide study. Heart 2000;83:425-8

10 Knight WB, Mee RBB. A cure for pulmonary arteriovenous fistulas? Ann Thorac Surg 1995:59:999-1001.

11 Castro M, Krowka M, Schroeder DR, et al. Frequency and clinical implications of increased pulmonary artery pressure in liver transplant patients. Mayo Clin Proc 1996;71:543-51.

12 Fallon MB, Abrams JA, Luo B, et al. The role of endothelial nitric oxide synthase in the pathogenesis of rat model of hepatopulmonary syndrome. Gastroenterology 1997;113:606-14.

13 Luo B, Abrams GA, Fallon MB. Endothelin-1 in the rat bile duct ligation model of hepatopulmonary syndrome: correlation with pulmonary dysfunction. J Hepatol 1998;29:571-78.

14 Williams A, Trewby P, Williams R, et al. Structural alterations to the pulmonary circulation in fulminant hepatic failure. Thorax 1979;34:447-53.

15 Mandell MS, Groves BM. Pulmonary hypertension in chronic liver disease. Clin Chest Med 1996;17:17-33.

16 Ashrafian $\mathrm{H}$, Swan L. The mechanism of formation of pulmonary arteriovenous malformations associated with the classic Glenn shunt (superior cavopulmonary anastomosis). Heart 2002;88:639.

17 Schenk P, Madl C, Rezaie-Maid S. Methylene blue improves the hepatopulmonary syndrome. Ann Intern Med 2000;133:701-6.

New Heart online submission and review system

We are pleased to inform authors and reviewers of Heart's new online submission and review system. Bench>Press is a fully integrated electronic system which uses the internet to allow rapid and efficient submission of manuscripts, and the entire peer review process to be conducted online.

Authors can submit their manuscript in any standard word processing software. Graphic formats acceptable are: .jpeg, .tiff, .gif, and .eps. Text and graphic files are automatically converted to PDF for ease of distribution and reviewing purposes. Authors are asked to approve their submission before it formally enters the reviewing process.

To access the system click on "SUBMITTING YOUR MANUSCRIPT" on the Heart homepage: http://www.heartinl.com/, or you can access Bench>Press directly at http://submit-heart.bmijournals.com/.

We are very excited with this new development and we would encourage authors and reviewers to use the on-line system where possible. It really is simple to use and should be a big improvement on the current peer review process. Full instructions can be found on Bench>Press and Heart online. Please contact Natalie Davies, Project Manager, ndavies@bmigroup.com for further information.

\section{Pre-register with the system}

We would be grateful if all Heart authors and reviewers pre-registered with the system. This will give you the opportunity to update your contact and expertise data, allowing us to provide you with a more efficient service.

\section{Instructions for registering}

1. Enter http://submit-heart.bmijournals.com

2. Click on "Create a New Account" in the upper left hand side of the Bench>Press homepage

3. Enter your email address in the space provided.

4. Choose a password for yourself and enter it in the spaces provided.

5. Complete the question of your choice to be used in the event you cannot remember your password at a later time (this will needed if you forget your password).

6. Click on the "Complete Step 10" button at the bottom of the screen.

7. Check the e-mail account you registered under. An email will be sent to you with a verification number and URL.

8. Once you receive the email, copy the verification number and click on the URL hyperlink. Enter the verification number in the relevant field. Click on 'Verify $\mathrm{Me}^{\prime}$. This is for security reasons and to check that your account is not being used fraudulently.

9. Enter/amend your contact information, and update your expertise data. 